\title{
LabVIEW-based control and acquisition system for the dosimetric characterization of a silicon strip detector
}

Cite as: Rev. Sci. Instrum. 88, 025104 (2017); https://doi.org/10.1063/1.4974817

Submitted: 24 January 2016 . Accepted: 11 January 2017 . Published Online: 02 February 2017

M. C. Ovejero, A. Pérez Vega-Leal, M. I. Gallardo, J. M. Espino (D), A. Selva, M. A. Cortés-Giraldo, and R. Arráns

\section{ARTICLES YOU MAY BE INTERESTED IN}

A simplified digital lock-in amplifier for the scanning grating spectrometer

Review of Scientific Instruments 88, 023101 (2017); https://doi.org/10.1063/1.4974755

Note: A temperature-stable low-noise transimpedance amplifier for microcurrent measurement

Review of Scientific Instruments 88, 026101 (2017); https://doi.org/10.1063/1.4974741

High resolution and stability roll angle measurement method for precision linear displacement stages

Review of Scientific Instruments 88, 023102 (2017); https://doi.org/10.1063/1.4974816

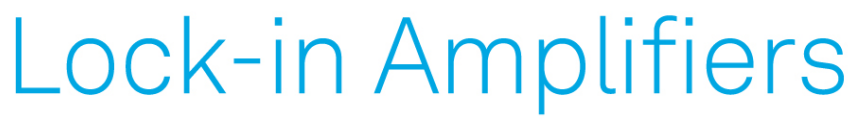

... and more, from DC to $600 \mathrm{MHz}$ Watch 


\title{
LabVIEW-based control and acquisition system for the dosimetric characterization of a silicon strip detector
}

\author{
M. C. Ovejero, ${ }^{1, a)}$ A. Pérez Vega-Leal, ${ }^{2}$ M. I. Gallardo, ${ }^{1}$ J. M. Espino, ${ }^{1}$ A. Selva, ${ }^{3}$ \\ M. A. Cortés-Giraldo, ${ }^{1}$ and R. Arráns ${ }^{4}$ \\ ${ }^{1}$ Departamento de Física Atómica, Molecular y Nuclear, Facultad de Física, \\ Universidad de Sevilla, Seville, Spain \\ ${ }^{2}$ Departamento de Tecnología Electrónica, Escuela de Ingenieros, Universidad de Sevilla, Seville, Spain \\ ${ }^{3}$ Dipartimento di Fisica e Astronomia "Galileo Galilei", 35131 Padova, Italy \\ ${ }^{4}$ Hospital Universitario Virgen Macarena, 41007 Seville, Spain
}

(Received 24 January 2016; accepted 11 January 2017; published online 2 February 2017)

\begin{abstract}
The aim of this work is to present a new data acquisition, control, and analysis software system written in LabVIEW. This system has been designed to obtain the dosimetry of a silicon strip detector in polyethylene. It allows the full automation of the experiments and data analysis required for the dosimetric characterization of silicon detectors. It becomes a useful tool that can be applied in the daily routine check of a beam accelerator. Published by AIP Publishing. [http://dx.doi.org/10.1063/1.4974817]
\end{abstract}

\section{INTRODUCTION}

Traditionally, the verification of complex photonradiotherapy treatments, like Intensity Modulated Radiation Therapy (IMRT), has been carried out by means of radiographic films, which are inserted in a water-equivalent phantom and irradiated by an accelerator. ${ }^{1}$ The radiographic films have to be developed and read with a scanner. The dose map is then obtained as a function of the optic density. The use of these films has some disadvantages such as the manipulation sensitivity and the time-consuming film development process. In addition, the increased use of digital radiometers is leading to the disappearance of films and development devices.

Despite these inconveniences, films have some advantages which until today could not have been achieved by any other method: ${ }^{2}$ first they provide excellent spatial resolution $(<<1 \mathrm{~mm})$ usually limited by their granular dimensions and the resolution of the scanner. Second they can measure dose maps in the axial plane, i.e., in the same plane in which the treatment planning is performed. Efforts are being made with many different technologies to develop new detection systems which will improve the traditional ones, guaranteeing optimum and safe conditions for the patient. Silicon detectors in particular are among these different new technologies. Some important projects developed by scientific collaborations involving silicon detectors are dedicated to medical applications such as MAESTRO, DOSI, and CMRP DMG detectors, ${ }^{3}$ and other alternative devices were developed by companies with their associated analysis software: MapCHECK, ${ }^{1,4}$ MATRIXX,,${ }^{5-7}$ and OCTAVIUS 729..$^{5,8}$

Within this context the Radia collaboration appears, ${ }^{2}$ whose main innovation is the development of the first

a)Electronic mail: movejero@us.es prototype of a system which measures the dose in the axial plane, with a monolithic silicon detector.

After a critical review of the first prototype, ${ }^{2}$ a new one $^{9}$ employing a Dual Single Sided Silicon Strip Detector (DSSSSD), also from Micron Semiconductors, Ltd., has been developed in order to improve its performance. The DSSSSD comprises two independent SSSSD segmented into 32 strips and mounted in a perpendicular configuration. ${ }^{9}$ Hence the use of this technology requires a high number of allocated channels and the development of a complex multichannel readout electronic system to measure the absorbed dose, integrating the total received charge in every strip.

For this second prototype, a new software instrument has been developed which not only manages the data acquisition and storage but also automates the entire process. This reduces errors and the time required for analysis. The time required to set-up, calibrate, and analyse the results with this second prototype is reduced to less than half an hour, thus creating a more efficient system, compared to the previous one.

This virtual instrument becomes the first software tool developed by a scientific collaboration, which allows the dosimetric characterization of silicon strip detectors. Furthermore, it can be applied in the daily routine commissioning of the beam accelerator.

\section{EXPERIMENTAL SETUP}

\section{A. The DSSSSD}

Micron Semiconductor, Ltd., built the Dual Single Sided Silicon Strip Detector (DSSSSD) BB7, using two commercial BB7-500 ${ }^{10}$ detectors placed in a special configuration, which is detailed in the next paragraph. Every BB7-500 is segmented on one side into 32 strips ( $2 \mathrm{~mm}$ wide) and a guard ring. The active area of every SSSSD is $64 \times 64 \mathrm{~mm}^{2}$ with a $500 \mu \mathrm{m}$ thickness of silicon. 


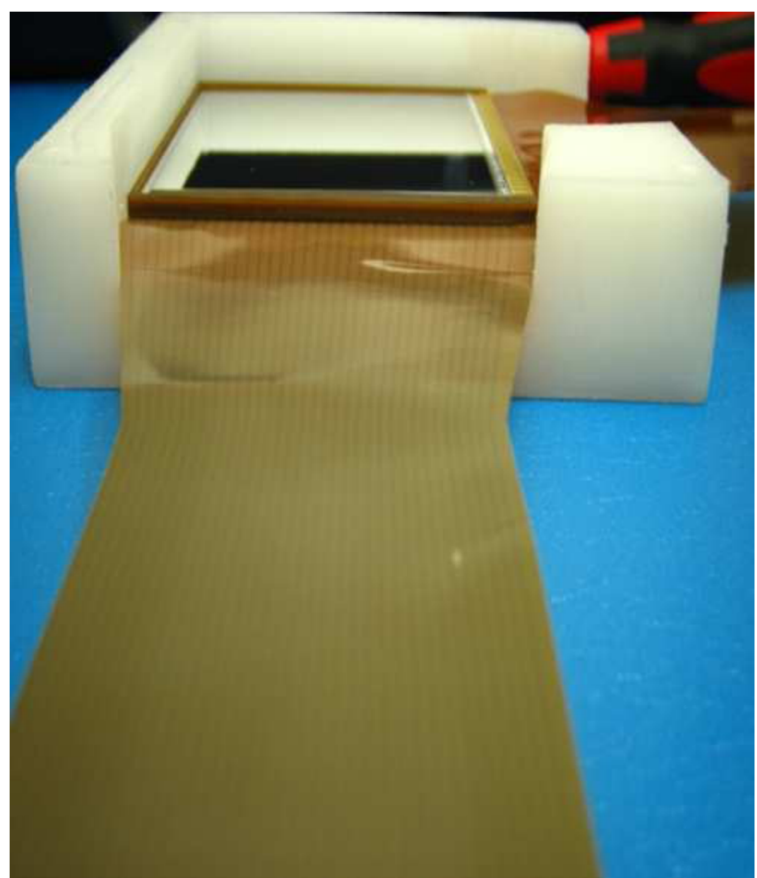

FIG. 1. The final version of the dual SSSSD chip for radiotherapy verification, placed in a polyethylene box, which is designed to be coupled to different phantoms.

The two BB7-500 detectors are mounted on parallel planes, with the strips arranged perpendicular to and between them, separated by a $500 \mu \mathrm{m}$ dielectric layer, of the same size as the silicon layer, minimizing the air gap in between (Fig. 1).

\section{B. The phantoms}

The DSSSSD is installed inside a polyethylene box specifically designed to house it with high precision. This box was constructed to house the detector in stable and safe conditions to minimize the air gap between the detecting volume and the wall of the box. In this way, the electronic equilibrium of the electrons which reach the surface of the detectors is maintained. The detector packed in its box is installed inside a slab phantom (Fig. 2) that was designed with the main purpose of characterizing the detector response, or within a cylindrical one (Fig. 3) in order to study the behaviour in the axial plane. ${ }^{2}$

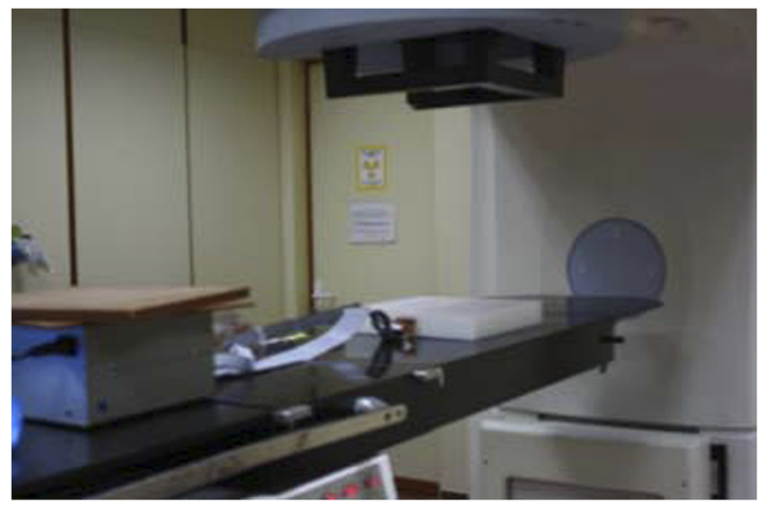

FIG. 2. Experimental setup with the detector housed in the slab phantom.

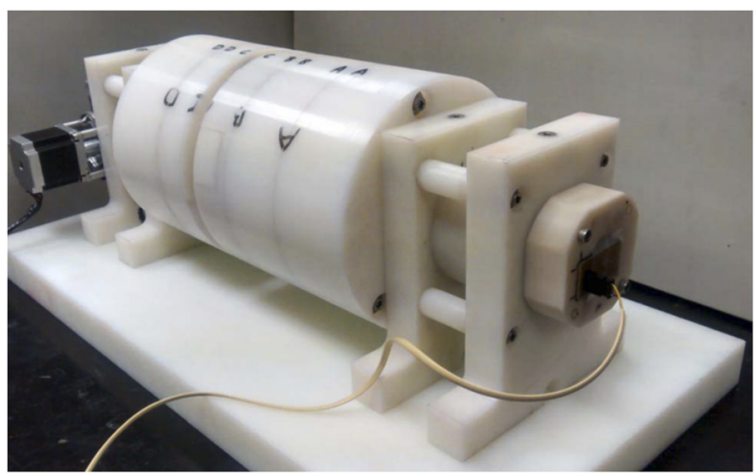

FIG. 3. The cylindrical phantom rotates around its central axis. The motor installed on one end of the axis and on the other side the angle sensor is placed on a PCB, which can be seen at the right side of the picture.

\section{The electronic control system}

A data acquisition (DAQ) and control system with 64 readout channels, 16-bits resolution, a selectable sample period from 100 to $500 \mathrm{~ms}$, and one inner digital trigger has been specifically designed at the Escuela Superior de Ingenieria, University of Seville (ESI-US) to read the charge generated in each strip of the detector.

Every strip of the silicon detector is connected to its own read-out channel in the DAQ system through an initial signal conditioning stage, built with a charge integration circuit. ${ }^{11}$ The output voltage, proportional to the charge, is digitalized with an analog to digital converter (ADC) and sent to a microprocessor that organizes the sampled data into the CAN format, which is the chosen communications protocol.

\section{The experiment}

The measurements for the characterization of the detection system have been carried out with medical linacs at the Virgen Macarena University Hospital in Seville (Fig. 2). The linac accelerates electrons which produce photons, when colliding with a Wolfram target by the Bremsstrahlung effect. The photon fields are shaped by means of a multileaf collimator (MLC) moving along the $\mathrm{X}$-axis, and jaws moving along the Y-axis, both systems installed in the accelerator's head. At therapy energy, photons interact with water equivalent material of the phantoms (or the human body tissue) mainly by the Compton interaction. ${ }^{12}$ Electrons set in motion by the photons hitting the phantom go through the detector, where they leave part of their energy, hence contributing to the absorbed dose.

Each strip of the DSSSSD is considered as an independent detector and each has its own electronic chain as earlier referenced, hence slight variations in resistance, capacitance, and amplifications occurring between one chain and another.

\section{E. Characterization}

To characterize the dosimetric response of the detector prior to obtaining dose maps for radiotherapy treatment verification, which is the final aim of the setup, several experiments 
must be carried out. These experiments involve irradiating the silicon detector located inside the specifically developed slab or cylindrical phantom with the linac within a range of 2-500 monitor units, depending on the characteristic under analysis. The monitor units are defined by the accelerator; in this case it is $3.33 \mathrm{MU} / \mathrm{s}$. Both phantoms are made with a water-equivalent material.

The slab phantom is used to obtain the standard dosimetric characterization of the detector. ${ }^{13}$ The cylindrical phantom is used to study the angular response of the detector. ${ }^{14}$ The response of the detector may change with the angle of incidence of the beam of the accelerator. To study its angular response, the cylindrical phantom is rotated, with the detector placed inside. When the detector is placed with the strips parallel to the accelerator beam, it is considered as the angle zero or the reference. This is the first step before the reconstruction of dose maps in the axial plane. The phantom should be rotated with an angular resolution of $5^{\circ}$; it is to run the $180^{\circ}$ for every strip of the 32 , in order to obtain the calibration of the detector in the axial plane.

The virtual instrument has been developed and tested for both scenarios. Fig. 2 shows the slab phantom which houses the detector. Within this phantom, the detector is irradiated by the clinical linac perpendicular to its active area. When the experiment is carried out with the detector hosted in the cylindrical phantom, besides allowing control of the DAQ, the LabVIEW interface can also control the rotation of the phantom (Fig. 3).

The digitalized data obtained by irradiating the detector, coming from the 64 channels of the DAQ, are transferred via a CAN bus. The PC-based LabVIEW application described in this paper reads the CAN frames with their origin in the DAQ and arranges them, keeping the synchrony in order to obtain the corresponding information from every strip of the detector.

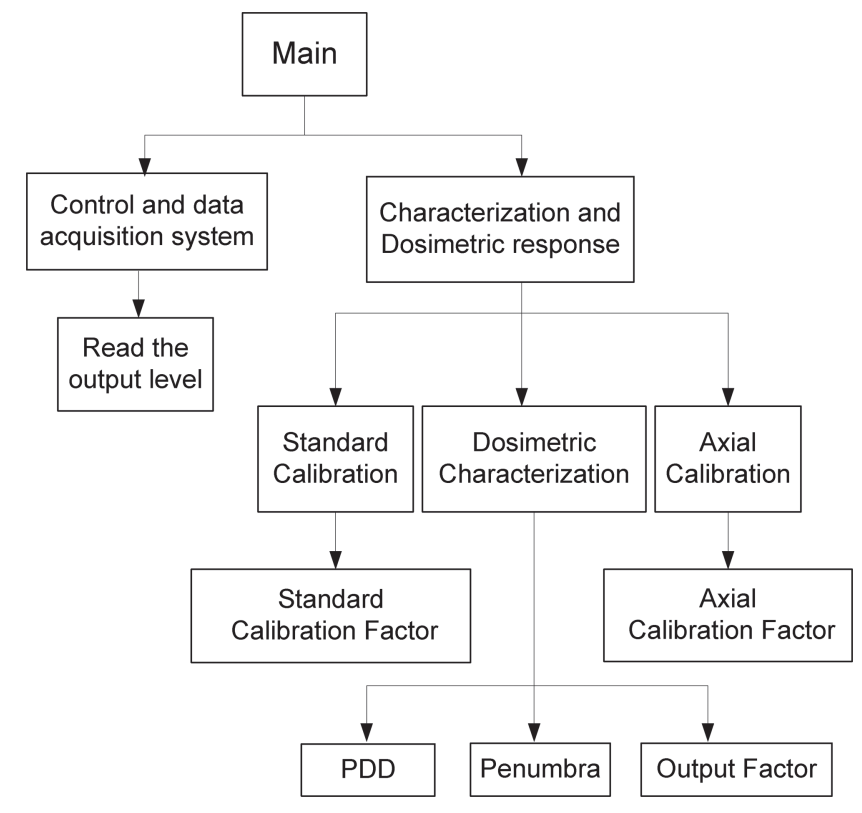

FIG. 4. SW architecture developed for the interface. From a main front panel, the user can reach the main functions.

\section{MEASUREMENTS AND DESCRIPTIONS OF THE LabVIEW SOFTWARE ARCHITECTURE}

A new LabVIEW tool has been developed to, in the first instance, automatically carry out measures and data analysis of a silicon strip detector. The LabVIEW software has been widely adopted for the data acquisition and control of customized scientific instrumentation. ${ }^{15-23}$

In order to get maintainable architecture, a structured modular code following a different level for each functionality has been implemented. The architecture of the software is shown in Fig. 4. From a main .vi two different functions can be activated: "Control and Data Acquisition System (CDAS)"

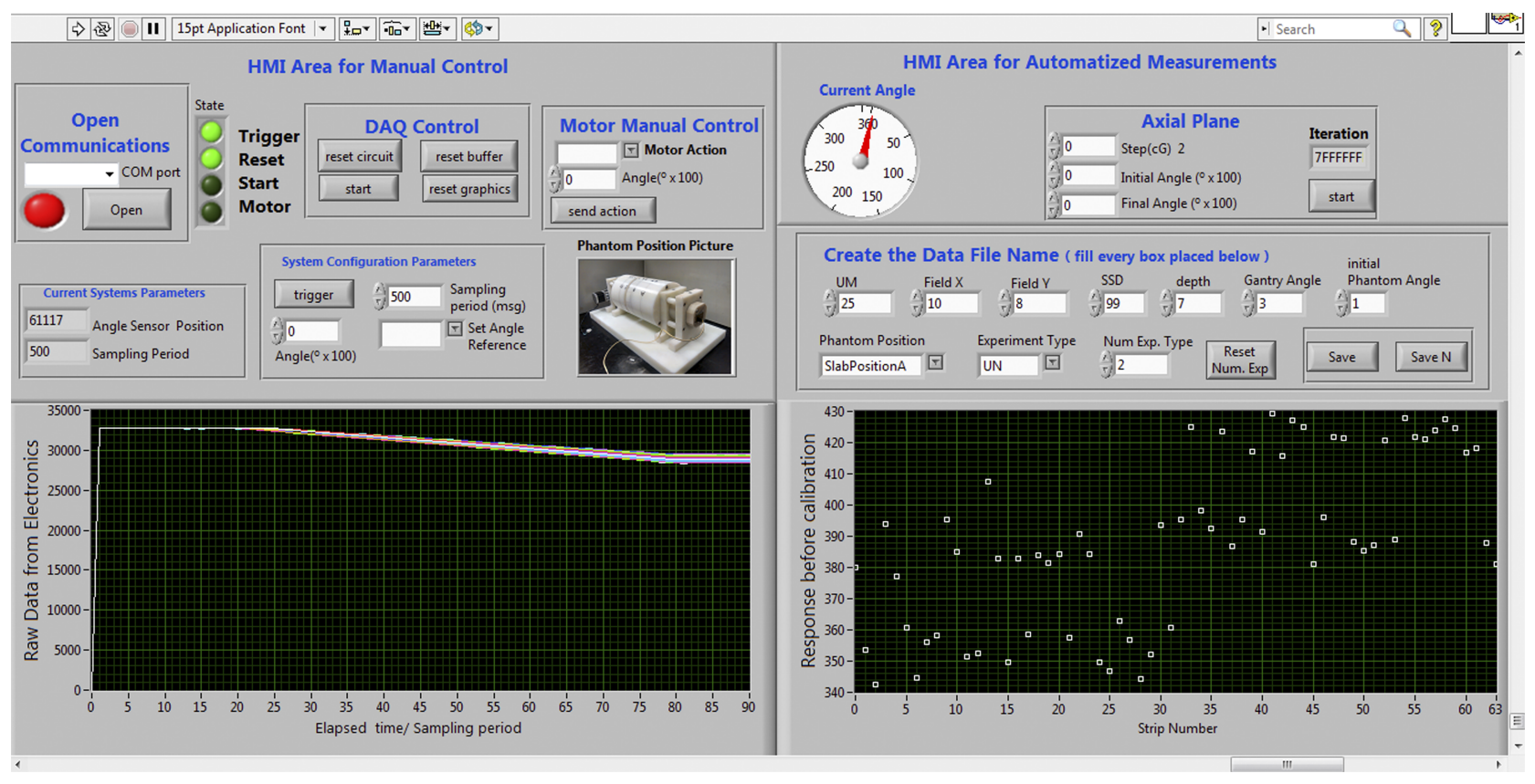

FIG. 5. Front panel for the data acquisition and automatic measurements in the axial plane. 
or "Characterization of the dosimetric response (CDR)." This architecture creates separated structures for hardware communication and programming functionalities, thereby ensuring that the system is more easily scalable.

\section{A. System initialization}

First we will focus on the CDAS window, designed for both of the previously described setups. It can be seen in Fig. 5 . The flowchart for manual experiments with the slab or the cylindrical setup can be carried out with the various controls and indicators placed on the upper left area. The input parameters required to carry out automatic measurements in the axial plane can be entered into the upper right area, according to the algorithms described in Sec III C.

The necessary operations required to start an acquisition are outlined in the flowchart Fig. 6. When the LabVIEW interface establishes the CAN bus communication, select the COM port and push the "OPEN" button.

Once the CAN bus has been opened, the LabVIEW interface is continuously checking if it has any data left to read from the data acquisition (DAQ) system. The read data have an incremental time-stamp index which starts when communications are established. The instant of time of every sample can

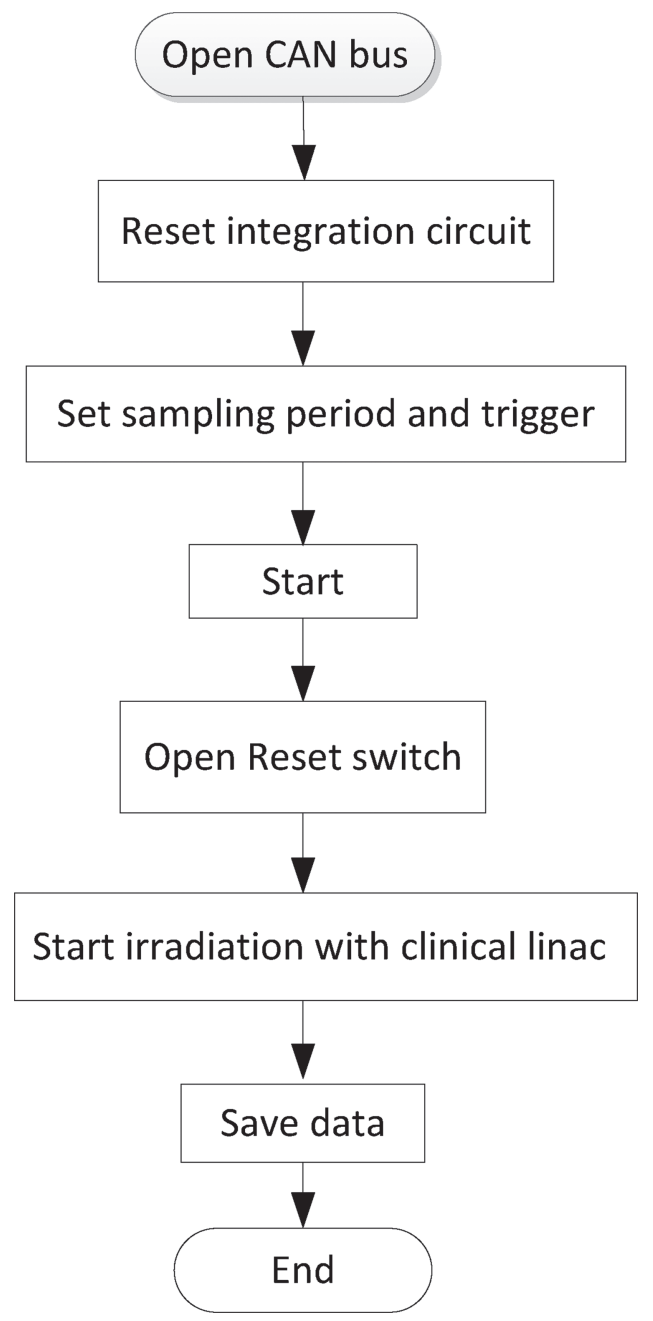

FIG. 6. Operations to be done in order to start an acquisition are outlined in this flowchart. be determined with reference to the time when the experiment was initiated. In order to have a time index (or time-stamp) related to the beginning of each irradiation, and not to the opening of the communication bus, a command to restart the time index of the sampled data can be sent through the "reset buffer" button.

Before starting the process of the irradiation of the detector, a reset command ("reset circuit" button) is sent from the interface to the integration circuit, in order to clear it of any residual charge which could distort the measured dose. Later, the user can set the sampling period and trigger the digitization of the signal ("trigger" button). Afterwards, the user can initiate the start command from the interface ("start" button).

\section{B. Measurement}

To start charge accumulation, the user sends the command that switches off the reset of the capacitors ("reset circuit").

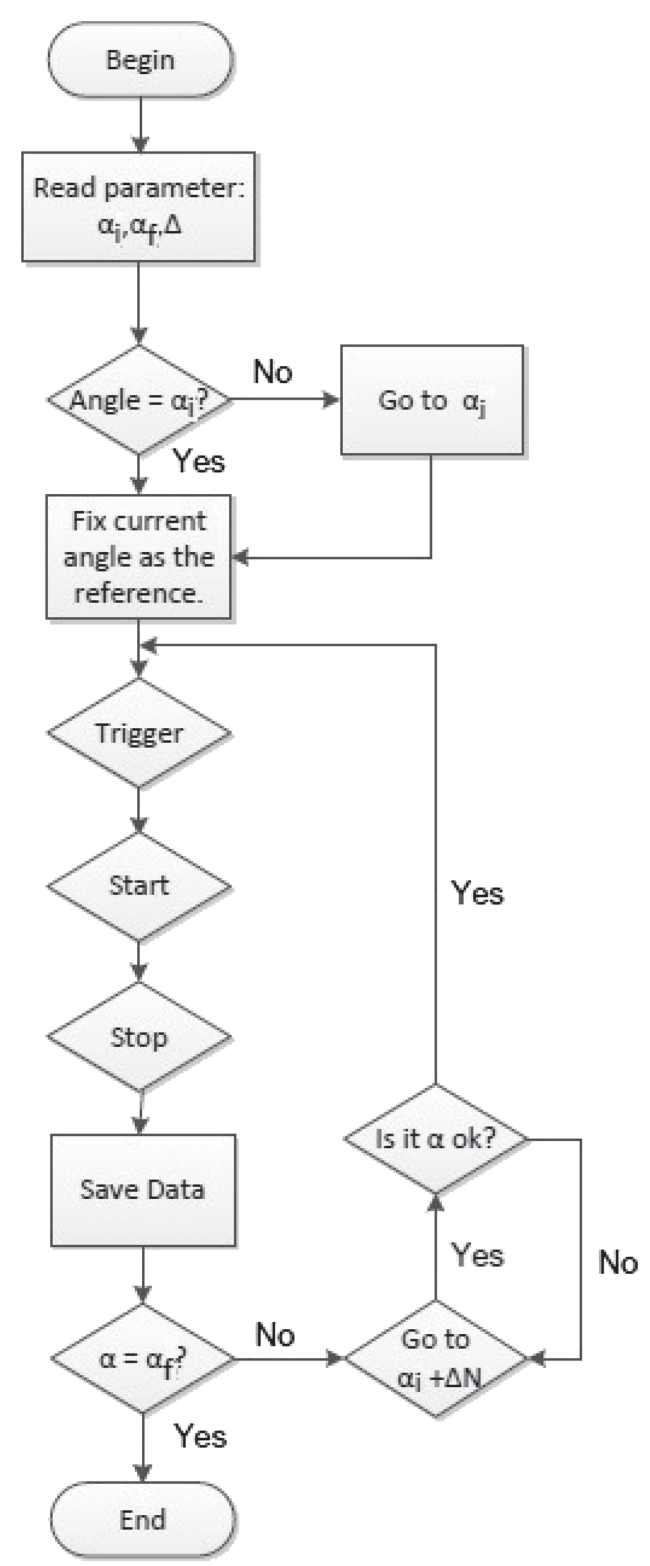

FIG. 7. Algorithm to automate the measurements in the axial plane. 
This should be done just before the linac starts the irradiation, in order to avoid the collection of spurious current. The bottom left area displays the output read by the LabVIEW interface from the DAQ when the detector is being irradiated. The bottom right area monitors the final digital reading for each strip. ${ }^{2,9}$ These data can be stored using the "Save" function which can be called up from the box above the plot. In order to save calibrated data, "Save N" must be used. Both types of sampled data are saved with their time-stamp.

In the case in which the detector is placed in the cylindrical phantom, the process of data acquisition follows the same steps already outlined for the slab phantom; the only difference is that prior to the start the angular position of the phantom must be identified and set. The angular reference must be defined and the configuration in which the strips are parallel to the beam axis is set as angle zero.

The LabVIEW interface sets the reference angle in the "system configuration parameters" area and moves the cylindrical phantom to a particular angular position through the "motor manual control" area.

To obtain the calibration factor in the axial plane which is necessary to obtain the $2 \mathrm{D}$ dose map of an entire treatment plan, an angular resolution of two degrees is required by the reconstruction algorithm. This is because the experiments in the axial plane require a high number of measurements at different angular positions, which would in turn require a high number of actions from the user.

In order to give the user the option of carrying out the acquisition automatically, a template has been developed:

\section{Automated measurements}

The computer automation of axial plane measurements follows the procedure outlined in Fig. 7, which is implemented as a sequential case structure. To start the acquisition, the required input parameters are the initial angle $\alpha_{i}$, the final angle $\alpha_{f}$, and the step $\Delta$. The LabVIEW sequence reads $\alpha_{i}$ and sends the detector to that angular position. Once that position has been reached, the procedure outlined in Fig. 6 is automatically executed. Then, once the data for that angle are automatically acquired and saved, the detector goes to the next angle (Eq. (1)),

$$
\alpha=\alpha_{i}+\Delta N,
$$

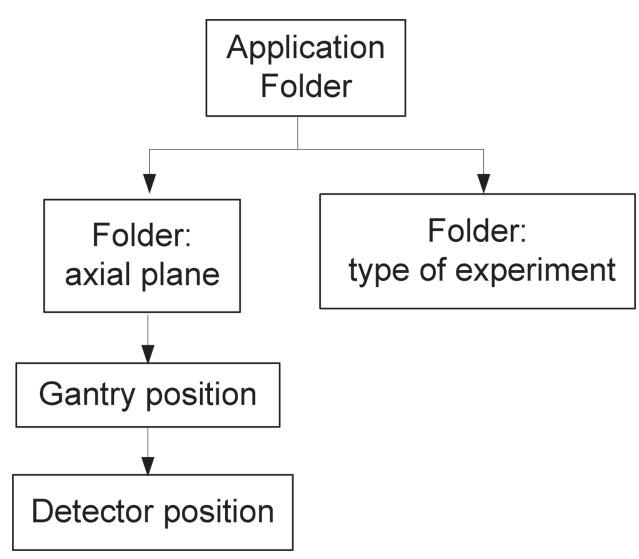

FIG. 8. Folder structure to arrange the information.

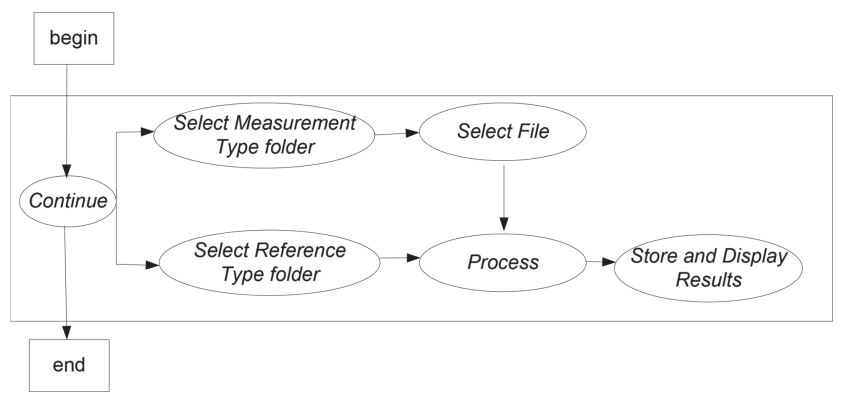

FIG. 9. Algorithm to automate the slab phantom analysis routines.

where

$$
N=\frac{\alpha_{f}-\alpha_{i}}{\Delta} .
$$

The algorithm continues until $\alpha=\alpha_{f}$.

The experiments are carried out for the different angular positions from the gantry as well. The gantry angle is kept fixed while the various angular measurements at different phantom angles are carried out. The different number of gantry angles is determined by the prescribed treatment.

\section{Data log and analysis}

Data are stored following the folder structure shown in Fig. 8. Every time the user activates the function "Save data" (press the button "Save"), a file is created whose name contains the values of the main parameters which have been set during the particular acquisition. The file is then stored within a specific folder created for that kind of measurement if it does not already exist. The file name for acquisitions with the slab phantom follows the structure "NumberOfMeasure, PhantomPosition, NumberOfMonitorUnits, FieldSize SourceSurfaceDistance, SolidWaterDepth," while the file for acquisitions with the cylindrical setup follows the structure "NumberOfMeasure, AngularPosition."

These structures automate the data analysis in order to obtain the dosimetric characterization:

Once the data are acquired with the detector placed in the slab phantom, in different configurations following procedures, the different parameters under study can be obtained: PDD curves, analysis of penumbra and dose profiles, output factor measurements, and dose calibration. ${ }^{13}$

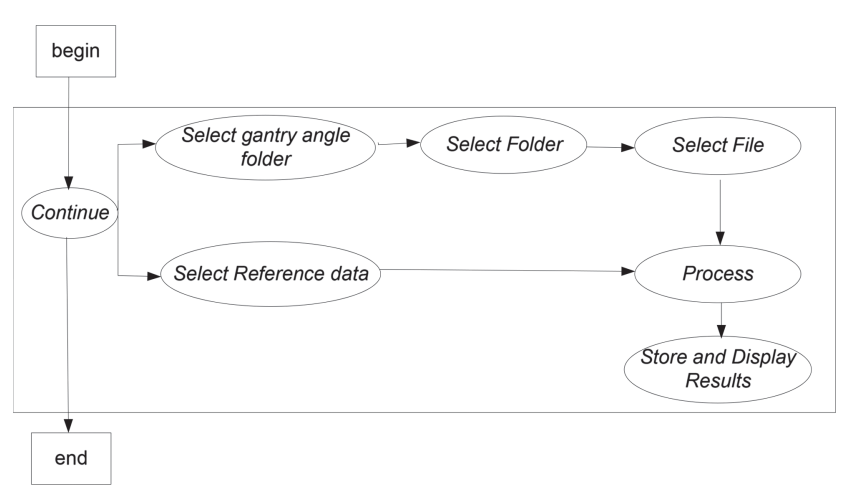

FIG. 10. Algorithm to automate the calculation of the calibration factor. 


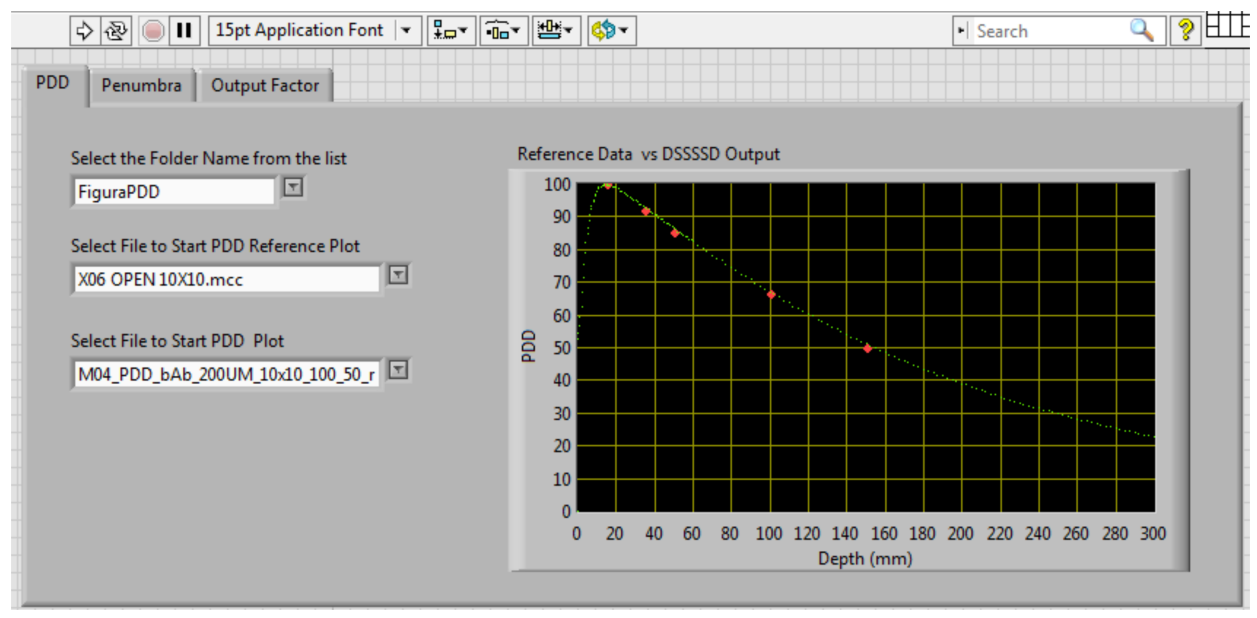

FIG. 11. Front panel with the reconstructed PDD curve. Big red dots correspond with measures taken with the DSSSSD and small green dots correspond with the ionization chamber measurements provided by the Hospital Universitario Virgen Macarena of Seville.

\section{E. Results presentation}

The result of every parameter in a different tab is accessed through the LabVIEW interface following the "CDR" tab from the main.

A template has been created for the automation of data analysis in order to obtain every parameter with both setups. Each of them has its corresponding tab in the front panel of a .vi. It has been implemented using an event structure. When the user selects the tab and searches for a file from within the defined architecture of files, data are processed and the result is displayed. The front panel acts as a user-friendly interface. The user selects the folder which contains the measured data and the reference data, following the algorithm of Fig. 9.

As an example, Fig. 10 shows the automated algorithm to obtain the calibration factor in the axial plane as a function of every angular position of the cylindrical phantom. The standard calibration factor is applied to data measured in the axial plane. If the measuring angle corresponds to a reference value of the hospital Treatment Planning System (TPS), the calibration factor for that angle is obtained as the ratio between the measured number of digital counts and the theoretical dose value provided by the TPS. The resulting values are stored in a file, within the same folder as the input data. In order for the data for every angle to be calibrated, the data file is multiplied by its axial plane calibration factor. Since no angular dependency is expected, this factor will be the same for every angle in our system. ${ }^{2}$ The calibrated data are stored in another file, adding the suffix "c" to the file name.

\section{EXAMPLE OF APPLICATION}

As an example of application, Fig. 11 shows the result for the PDD curve ${ }^{13}$ obtained with the DSSSSD housed in the slab phantom using the LabVIEW tool.

Several measurements to study the PDD are carried out with the DSSSSD at different depths. The detector is placed perpendicular to the beam position at a solid water depth of $1.5,3.5,5,10$, and $15 \mathrm{~cm}$. These measurements are compared with those taken with an ionization chamber at the Hospital
Virgen Macarena for $(10 \times 10) \mathrm{cm}^{2}$ field, whose values are also normalized to the maximum of the profile, placed at a depth $\mathrm{d}=1.5 \mathrm{~cm}$. We can see that both measurements are consistent, within error bars.

Small green dots represent the selected reference file; in this case an ionization chamber measurement provided by the Hospital Universitario Virgen Macarena of Seville. Big red dots represent the measurements of the DSSSSD, for different solid water depths.

\section{DISCUSSION AND CONCLUSION}

We have presented the results for implementing a LabVIEW based software for the data acquisition and analysis from which the dosimetric characterization of a silicon strip detector can be determined. It has been developed and tested and the first experimental results obtained with a new detection prototype have been analyzed.

This tool which was initially developed specifically for our system can easily be adapted for another kind of detector. It can be used to obtain the dosimetric characterization for any detector, as long as the input and reference data are placed in a folder that matches the input format required by the analysis algorithms. This tool is also the first step towards the development of a software which allows dosimetric characterization and dose map reconstruction, following the clinical quality assurance norms for radiotherapy verification.

This LabVIEW software integrates the CDAS and the CDR in the same environment. Thanks to the automated templates making it time efficient to the user. This is the first LabVIEW tool which allows the dosimetric characterization of a silicon strip detector.

\section{ACKNOWLEDGMENTS}

This work is supported by Spanish Research Project Nos. FPA2013-47327-C2-1-R and FPA2014-53290-C2-2P; by Andalusian Research Project No. P12-FQM-1605; by Radia 2 Contract No. 68/83-Ref. 0214/0129; and by Consolider-Ingenio 2010 Project No. CSD2007-004. 
M. C. Ovejero would like to thank Dr. Agnes Nasozy Kamya for her angelical patient editing of the final English version.

${ }^{1}$ X. R. Zhu, P. A. Jursinic, D. F. Grimm, F. Lopez, J. Rownd, and M. Gillin, "Evaluation of kodak EDR2 film for dose verification of intensity modulated radiation therapy delivered by a static multileaf collimator," Med. Phys. 29, 1687-1692 (2002).

${ }^{2}$ A. Bocci, M. C. Giraldo, M. I. Gallardo, and J. M. Espino, "Silicon strip detector for a novel 2D dosimetric method for radiotherapy treatment verification," Nucl. Instrum. Methods Phys. Res., Sect. A 673, 98-106 (2012).

${ }^{3}$ S. Manolopoulos, C. Buttar, M. Homer, and S. Martin, " $\Delta$ OSI: A prototype microstrip dosimeter for characterization of medical radiotherapy and radiosurgery systems," Nucl. Instrum. Methods Phys. Res., Sect. A 563, 229-232 (2006).

${ }^{4}$ Sun Nuclear Corp, available on-line at, http://www.sunnuclear.com/ medPhys/patientqa/mapcheck2/mapcheck2.asp, 2013.

${ }^{5} \mathrm{E}$. Spezi et al., "Characterization of a $2 \mathrm{D}$ ion chamber array for the verification of radiotherapy treatments," Phys. Med. Biol. 50, 3361 (2005).

${ }^{6} \mathrm{~J}$. Bedford, Y. Lee, P. Wai, and C. South, "Evaluation of the Delta 4 phantom for IMRT and VMAT verification," Phys. Med. Biol. 54, 167-176 (2009).

${ }^{7}$ IBA Dosimetry, available on-line at, http://www.iba-dosimetry.com/, 2013. ${ }^{8} \mathrm{PTW}$, available on-line at, http://www.ptw.de/octavius.html, 2013.

${ }^{9}$ M. A. G. Alvarez, Z. Abou-Haid, R. Arrans, M. C. Battaglia, M. A. C. Giraldo, J. M. E. Navas, M. I. Gallardo, M. Ovejero, A. P. Vega-Leal, A. Selva, and S. Walsh, "Novel dual single sided silicon strip detector chip for radiotherapy verification," in Proceedings of Science X LASNPA, 2013.

${ }^{10}$ See http://www.micronsemiconductor.co.uk/pdf/bb.pdf for "Micronsemiconductor, Specialist Detector for Nuclear Physics" (accessed 26 November, 2014).

${ }^{11}$ M. P. García and J. C. A. Anton, Instrumentación Electrónica (Thomson Editores, 2004).
${ }^{12}$ G. F. Knoll, Radiation Detection and Measurements (Wiley, 2010), Chap. 10.

${ }^{13}$ M. C. Ovejero, Z. Abou-Haidar, M. A. G. Alvarez, R. Arrans, C. Battaglia, M. A. Cortés-Giraldo, J. M. Espino, and M. I. Gallardo, "First results with a new detection system for complex radiotherapy treatment verification," Radiother. Oncol. 110, S71 (2014).

${ }^{14}$ P. A. Jursinic, "Angular dependence of dose sensitivity of surface diodes," Med. Phys. 36, 2165 (2009).

${ }^{15}$ J. Agraz, A. Grunfeld, D. Li, K. Cunningham, C. Willey, R. Pozos, and S. Wagner, "LabVIEW-based control software for para-hydrogen induced polarization instrumentation,” Rev. Sci. Instrum. 85, 044705 (2014).

${ }^{16}$ A. A. Topalov, I. Katsounaros, J. C. Meier, S. O. Klemm, and K. J. J. Mayrhofer, "Development and integration of a LabVIEW-based modular architecture for automated execution of electrochemical catalyst testing," Rev. Sci. Instrum. 82, 114103 (2011).

${ }^{17}$ J. Ballesteros, J. I. F. Palop, M. A. Hernndez, R. M. Crespo, and S. B. del Pino, "LabVIEW virtual instrument for automatic plasma diagnostic," Rev. Sci. Instrum. 75, 90-93 (2004).

${ }^{18} \mathrm{~B}$. Ashcroft and T. Oosterkamp, "Automicromanager: A microscopy scripting toolkit for LabVIEW and other programming environments," Rev. Sci. Instrum. 81, 113708 (2010).

${ }^{19} \mathrm{~S}$. Owen and D. Hall, "Fast line-based experiment timing system for LabVIEW,” Rev. Sci. Instrum. 75, 259265 (2004).

${ }^{20}$ J. Zhao, Z. Zhao, L. Du, D. Geng, and S. Wu, "Photoacoustic detection of $\mathrm{CO}_{2}$ based on LabVIEW at $10.303 \mu \mathrm{m}$," Rev. Sci. Instrum. 82, 044904 (2011).

${ }^{21}$ J. Bok and P. Schauer, "LabVIEW-based control and data acquisition system for cathodoluminescence experiments," Rev. Sci. Instrum. 82, 113109 (2011).

${ }^{22}$ D. J. Kim and Z. Fisk, "A LabVIEW based template for user created experiment automation,” Rev. Sci. Instrum. 83, 123705 (2012).

${ }^{23}$ Y. Cuiand and L. Hanley, "Chims: Open-source instrument control software platform on LabVIEW for imaging/depth profiling mass spectrometers," Rev. Sci. Instrum. 86, 065106 (2015) 